\title{
Résumé de la conférence de Patrick Boucheron tenue à Francfort le 23 mai 2012
}

Patrick Boucheron

\section{(2) OpenEdition}

1 Journals

Édition électronique

URL : http://journals.openedition.org/ifha/395

DOI : 10.4000/ifha.395

ISSN : 2198-8943

Éditeur

IFRA - Institut franco-allemand (sciences historiques et sociales)

Édition imprimée

Date de publication : 30 septembre 2012

Pagination : 64-67

ISSN : 2190-0078

\section{Référence électronique}

Patrick Boucheron, « Résumé de la conférence de Patrick Boucheron tenue à Francfort le 23 mai 2012

», Revue de l'IFHA [En ligne], 4| 2012, mis en ligne le 14 février 2013, consulté le 15 septembre 2020

URL : http://journals.openedition.org/ifha/395

Ce document a été généré automatiquement le 15 septembre 2020.

(CIFHA 


\title{
Résumé de la conférence de Patrick Boucheron tenue à Francfort le 23
} mai 2012

\author{
Patrick Boucheron
}

\section{NOTE DE L'ÉDITEUR}

\section{Rapport établi par Patrick Boucheron}

La Renaissance italienne a-t-elle inventé l'urbanisme moderne? La question peut sembler presque incongrue, tant il apparaît clair aujourd'hui que les réalisations architecturales du XVe siècle à Florence, Venise ou Milan, mais aussi, et peut-être surtout, la théorisation de l'art de bâtir par Francesco di Giorgio Martini, Leon Battista Alberti ou Filarete, inaugurent notre propre souci de l'aménagement urbain. Pourtant, les villes ne se transforment guère au XVe siècle: au temps de la croissance démographique bloquée, il s'agit d'abord d'en conserver la forme, et le cas vénitien montre sans doute de manière exemplaire que cette idée de conservation de la ville a d'abord et avant tout une dimension politique. Ainsi serait-on tenté d'opposer un temps où la ville se construit sans être pensée (la période communale) et un temps où on la pense, sans la construire (la fin du Moyen Âge). Cette dichotomie est évidemment trop sommaire: on cherchera donc à évaluer l'apport véritable de la révolution albertienne dans l'art de bâtir, qui consiste sans doute à faire de l'architecture l'un des modes de la persuasion politique. Si l'architecture humaniste ne construit guère de villes, au moins construit-elle la figure sociale de l'architecte. Encore doit-on sans doute distinguer entre plusieurs échelles de transformations urbaines, et plusieurs moments du temps politique : ainsi proposera-t-on, dans un troisième et dernier temps, de réfléchir sur la manière dont les princes ont voulu, à Milan, à Mantoue ou à Urbino, transformer d'anciennes cités communales en villes princières. Ce qui supposait aussi d'agir sur la mémoire de la ville : car bâtir n'est pas seulement aménager l'espace, c'est 
aussi conserver et transformer le temps - le temps de la mémoire et le temps du politique.

1- Par ses dimensions, sa précision, sa technique, son réalisme, sa valeur documentaire, la vue de Venise gravée par Jacopo de' Barbari en 1500 est une œuvre exceptionnelle et inaugurale dans l'histoire de la représentation des villes. Il ne s'agit pas d'un plan, mais d'une veduta, d'une vue cavalière, en perspective, et à vol d'oiseau. Comme l'avait remarqué Pierre Francastel, on a peint les villes à vol d'oiseau bien avant de pouvoir les survoler réellement, et ce fait d'évidence doit nous permettre de mesurer la stupéfaction émerveillée de ceux qui voyaient une telle image. On tente ici de décrire l'aventure technologique que constitue l'impression d'une telle image de la ville, indissociable de la présence des imprimeurs allemands à Venise.

Ce que donne à voir le plan de Jacopo de' Barbari est d'abord ceci: en 1500, l'urbanisation de la ville est terminée - et si ce n'est les grandes églises baroques et leurs alentours immédiats, la forme de la ville est celle que l'on peut encore visiter aujourd'hui. Ce que Le Corbusier appelait «l'événement urbanistique » est accompli : Venise est la ville la plus minérale que l'on puisse imaginer, alors qu'elle est " assise dans l'eau ", comme dit Commynes, c'est-à-dire qu'elle se développe sur un site qui est tout sauf destiné au développement urbain. Ville sans sol, sans contado, et aussi sans muraille, Venise s'inscrit dans une géographie lagunaire qui est l'horizon de la représentation de Barbari. Cette veduta doit, pour être comprise, être confrontée à un événement énonciatif : l'essor, dans les documents de la pratique, d'un discours des périls, qui justifie de nouveaux travaux d'aménagements urbains.

On doit évidemment s'interroger sur l'effet de discours. À quoi sert-il ? À justifier une intervention croissante de l'État, sans doute. C'est en grande partie une reconstruction historiographique que de considérer que la construction de la ville entraîna, d'emblée, un accroissement de la puissance publique. Elisabeth Crouzet-Pavan a reconstitué la complexité initiale de l'entreprise de bonification vénitienne, conduite par la commune, mais aussi des associations de quartiers, des fondations religieuses, des nobles. Ce que donne à voir et à comprendre le discours de la conservation urbaine, c'est bien la politisation de l'art de bâtir dont Alberti sera plus tard le théoricien.

2- De ce point de vue, l'architecture connaît la même mutation que la peinture : pour le dire de manière très brève, le passage d'un art de la mémoire à un art de la persuasion. Poursuivant les intuitions de Daniel Arasse, les historiens de la perspective ont décrit ce passage d'un système mnémonique qui reflète l'ordre immuable et hiérarchisé du cosmos, où chacun est dans son locus et où les loci sont simplement juxtaposés, à un système rhétorique où les personnages se déplacent dans un espace commun et où la storia doit convaincre le spectateur. Mais ce qui chez Alberti vaut pour l'espace pictural vaut aussi pour l'espace urbain en tant que l'un et l'autre sont, fondamentalement, des espaces politiques. Du De pictura au De re aedificatoria circulent les mêmes concepts de modération et de persuasion, d'espace commun et de récit unifié, qui tous ont pour origine l'œuvre politique et morale de l'humaniste.

Voici pourquoi le De re aedificatoria est bien un livre politique, et pas seulement un traité pratique. Leon Battista Alberti lui a donné ce titre intriguant, usant, en latiniste subtil, d'un adjectif rare et n'existant pas dans la langue classique, aedificatorius, désignant le fait d'édifier. Au fond, ce qu'a écrit Alberti est bien un texte qui tente de comprendre, pour paraphraser Pierre Bourdieu, "ce que bâtir veut dire ». Le livre 
d'Alberti délivre un discours moral de la modération : il s'agit pour lui de définir un lexique architectural qui exprime sans ambiguïté la typologie des régimes politiques.

Lorsqu'il conseille au prince éclairé d'édifier un palais plutôt qu'une citadelle, l'humaniste agit de la même manière que le grammairien : il n'édicte pas des normes, mais note des usages - même si, en les notant, il contribue à les figer en règles. Le De re aedificatoria enregistre donc la grammaire du langage architectural qui avait cours dans l'Italie du Quattrocento. Or, ce langage, pour être efficace, doit être comme la langue vernaculaire : compris par tout le monde. Alberti affirme qu'il existe une disposition innée à saisir la beauté d'un édifice : « on peut s'en étonner et se demander pourquoi la nature nous fait immédiatement sentir à tous, ignorants comme savants, ce qu'il y a de juste ou de vicieux dans la conception des choses et dans leur exécution, en particulier dans ces questions où le sens de la vue l'emporte en acuité sur tous les autres ». Cette beauté s'impose et intimide, elle est proprement désarmante. C'est une protection contre la violence, puisqu'elle rend les choses inviolables. «Or, la beauté obtiendra, même de la part d'ennemis acharnés, qu'ils modèrent leur courroux et consentent à la laisser inviolée ». On doit prendre cette remarque très au sérieux, puisqu'elle rend compte de l'efficacité proprement politique que les princes attendaient, non seulement de la construction édilitaire, mais de l'embellissement des villes: ce pouvoir d'intimidation d'une beauté proprement désarmante fait de la mise en beauté du cadre urbain une sorte de mise en défense.

3- Plutôt que de suivre ces lignes de fuite, mieux vaut retourner au centre, dans ces grandes villes que l'architecture princière peine à transformer, pour mesurer l'apport véritable de la pensée humaniste dans la théorie et la pratique de la création urbaine. Celle-ci s'observe à deux niveaux : en deçà de la cité, celui de portions d'espace urbain réaménagées comme des villes idéales; au-delà de la cité, dans la théorisation d'une agglomération affranchie de la forme murée des cités médiévales.

À partir des années 1470 environ, les architectes décrivent des villes idéales libérées du modèle médiéval de la cité fortifiée. Eux qui passent leur temps à construire des fortifications rêvent d'une civilisation idéale et pacifiée où point n'est besoin de se garder de ses ennemis. Dans ses écrits, Alberti prône une ville ouverte, en expansion, qui peut déployer sans contrainte son emprise sur les campagnes environnantes. La ville idéale du Quattrocento se définit donc par opposition à la cité de Dante, retranchée à l'intérieur de ses vieilles murailles comme une lointaine vision d'un passé patriarcal.

Ce que la pensée urbaine rejette, au fond, c'est précisément la valeur idéologique que la civilisation communale avait investie dans l'enceinte urbaine: idéal de paix et de stabilité, de retranchement et de clôture. Au total, le moment humaniste est peut-être fondateur dans l'habitus des architectes, qui se rêvent créateurs de villes. Mais il constitue surtout un moment singulier, clos sur lui-même, presque une parenthèse.

Ce n'est peut-être pas lorsqu'ils rêvaient de cités idéales, ou même lorsqu'ils les réalisaient à échelle réduite, que les architectes humanistes ont le plus contribué à la constitution d'une théorie moderne de la création urbaine. L'utopie est au futur. La ville, elle, est toujours au présent. Mais elle juxtapose dans une contemporanéité plusieurs fragments de temporalités différentes, car elle est faite de ces formes ayant survécu à leur fonction. Cette thématique, qui a été décrite par Bernard Lepetit, n'est pas étrangère à la pensée humaniste, qui fut le cadre de l'invention du patrimoine. La gestion des usages sociaux de la ville, et le réinvestissement des hauts-lieux de la mémoire d'une ville par la présence du prince, constituent les enjeux essentiels de 
l'architecture humaniste. Gérer et embellir des organismes urbains, renouveler et régénérer la ville, plutôt que de fonder des villes nouvelles, fut une préoccupation constante. Car si la durée d'une ville est le territoire de l'historien, c'est aussi le chantier de l'architecte. 